ARTICLE

\title{
Large Scale Numerical Simulation for Superfluid Turbulence
}

\author{
Narimasa SASA and Masahiko MACHIDA \\ Japan Atomic Energy Agency, 6-9-3 Higashiueno, Taito-ku, Tokyo, 110-0015, Japan
}

\begin{abstract}
Large scale numerical simulation of quantum turbulence is performed by using 3-D time-dependent Gross-Pitaevskii equation. The energy spectrum obeying Kolmogorov law and large scale self-similar structure of quantum vortex tangle are found in a fully developed dumped turbulent state. Width of the inertial range becomes large depending on the system size of the simulation that is consistent with the result of the normal fluid turbulence. On the other hand, bottleneck effect near coherent length prevents the inertial range from extending to smaller scale.
\end{abstract}

KEYWORDS: numerical simulation, superfluid turbulence, Gross-Pitaevskii equation, Kolmogorov law, inertial range

\section{Introduction}

Recently, there has been a great interest in research of superfluid turbulence which consists of a tangle of quantized vortex lines with a fixed core radius and a quantized velocity circulation. ${ }^{1)}$ The superfluid has zero viscosity, and in the zero-temperature limit, the superfluid turbulence's Reynolds number is infinite. This brings (at least, the zero-temperature) superfluid turbulence to a desired prototype for better insight in the classical normal fluid turbulence.

The numerical study of superfluid turbulence by the Gross-Pitaevskii equation has been reported in a few papers. Nore et al. ${ }^{2)}$ solved the Gross-Pitaevskii equation with resolutions up to $512^{3}$ and observed that as the quantized vortices became tangled, the incompressible kinetic energy spectra seemed to obey the Kolmogorov-1941 (K41) law for a short period of time, but eventually deviated from it. Kobayashi and Tsubota ${ }^{3}$ solved the Gross-Pitaevskii equation on $256^{3}$ grid with an extra dissipation term at small scales. Yepez et al. ${ }^{4)}$ simulated the Gross-Pitaevskii equation on grids up to $5,760^{3}$ by using a unitary quantum lattice gas algorithm. They also found a spectrum $\mathrm{E}(\mathrm{k})$ and interpreted it as the K41 law of normal fluid turbulence. However, due to the choice of initial conditions, their simulation should correspond to the pure Kelvin waves region, thus supporting the L'vov-Nazarenko spectrum of Kelvin waves. ${ }^{5}$

In this paper, we numerically solve the Gross-Pitaevskii equation on numerical grid size from $256^{3}$ to $2,048^{3}$. First, we confirmed the K41 law in the normal fluid-region of about two decades long, which is wider than that of any previous work. Second, the visualization of vortices clearly shows the bundle-like structure, which has never been confirmed in the Gross-Pitaevskii simulations on smaller grids. Third, we find a plateau in the crossover region which is a clear manifestation of the energy stagnation.")

*Corresponding author, E-mail: sasa.narimasa@jaea.go.jp

(C) 2011 Atomic Energy Society of Japan, All Rights Reserved.

\section{Theoretical Formulation}

\section{Equation of Motion}

Time evolution of superfluid turbulence is described by the Gross-Pitaevskii equation,

$$
i \hbar \frac{\partial}{\partial t} \Psi(\mathbf{r}, t)=\left[-\frac{\hbar^{2}}{2 m} \nabla^{2}+g|\Psi(\mathbf{r}, t)|^{2}\right] \Psi(\mathbf{r}, t)
$$

The macroscopic wave function $\Psi(\mathbf{r}, t)(=\mathrm{R}(\mathbf{r}, \mathrm{t}) \exp [\mathrm{i} \theta(\mathbf{r}, \mathrm{t})])$ plays a role of the complex order parameter, and $g$ is the coupling constant. We define the velocity field $\mathbf{v}$ as

$$
\mathbf{v} \equiv \frac{\hbar}{\sqrt{2 m}}|\Psi(\mathbf{r}, t)| \nabla \theta(\mathbf{r}, t)=\mathbf{v}^{\mathbf{c}}+\mathbf{v}^{\mathbf{i}}
$$

and decompose it into the compressible part $\mathbf{v}^{\mathrm{c}}$ and the incompressible part $\mathbf{v}^{\mathrm{i}}$ where they satisfy the condition $\operatorname{rot}\left(\mathbf{v}^{\mathbf{c}}\right)=0$ and $\operatorname{div}\left(\mathbf{v}^{\mathrm{i}}\right)=0$, respectively. The incompressible part of the kinetic energy is given as

$$
E_{\text {kin }}^{\mathbf{i}}=\frac{1}{N_{\text {tot }}} \int\left(\mathbf{v}^{\mathbf{i}}\right)^{2} d \mathbf{x}\left(N_{t o t} \equiv \int|\Psi(\mathbf{r}, t)|^{2} d \mathbf{x}\right)
$$

and the spectrum of the incompressible kinetic energy $\mathrm{E}_{\mathrm{kin}}^{\mathrm{i}}(\mathrm{k}, \mathrm{t})$ is defined as

$$
E_{k i n}^{\mathbf{i}}(t)=\int E_{k i n}^{\mathbf{i}}(k, t) d k
$$

\section{Initial State}

As an initial state of our numerical simulation, we firstly divide each axis into equal 8 regions, i.e., the whole calculation domain is divided into $8^{3}$ cubes. On each apexes of cubes, the phase $\theta(\mathbf{r}, 0)$ are chosen to be random numbers between $-\alpha N \pi$ and $\alpha N \pi$ where $N$ is the mesh number in 1 -dimension and $\alpha=1 / 256$. Phase of every lattice points are obtained by interpolating the phase of the apexes cyclically in each direction. Absolute value of every lattice points are given as $|\Psi(\mathbf{r}, 0)|=1$. 


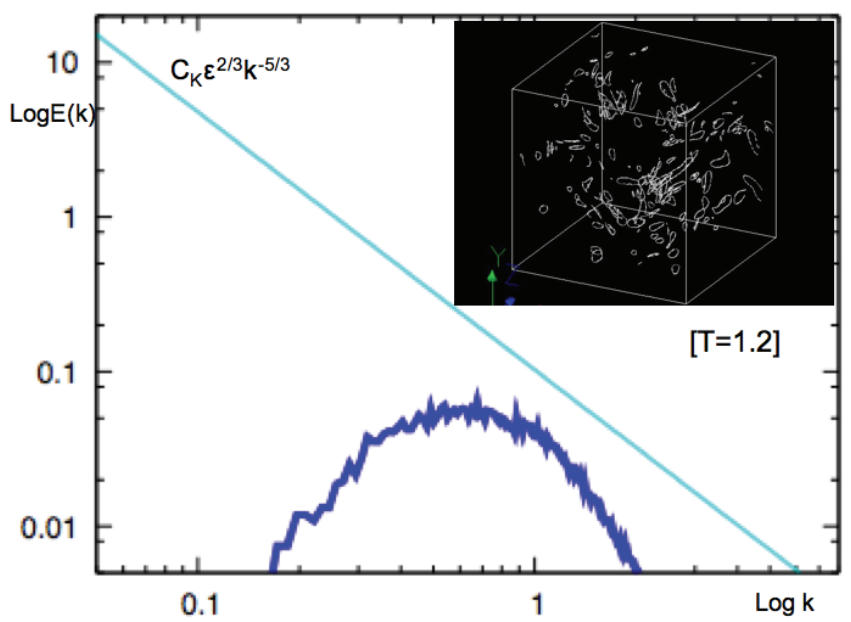

(a) $\mathrm{T}=1.2$

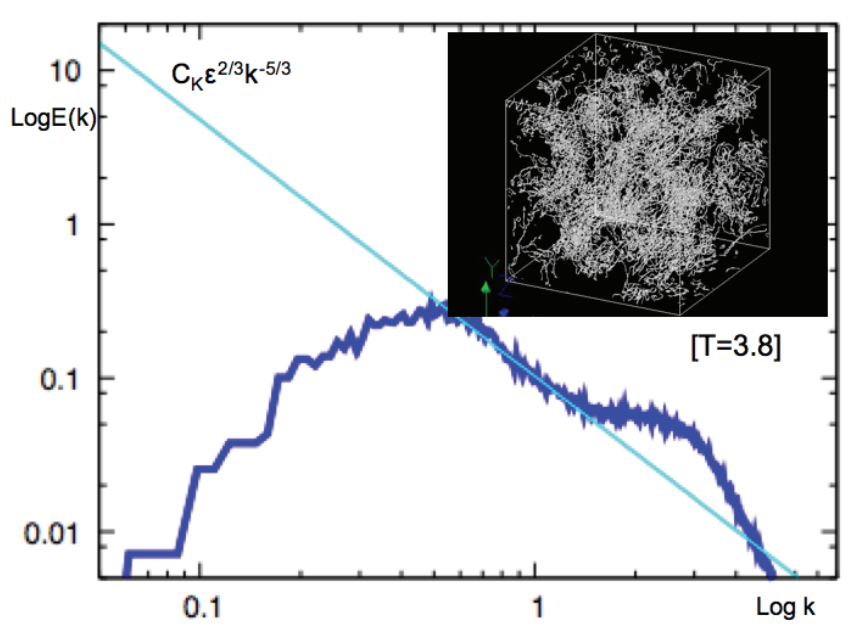

(b) $\mathrm{T}=3.8$

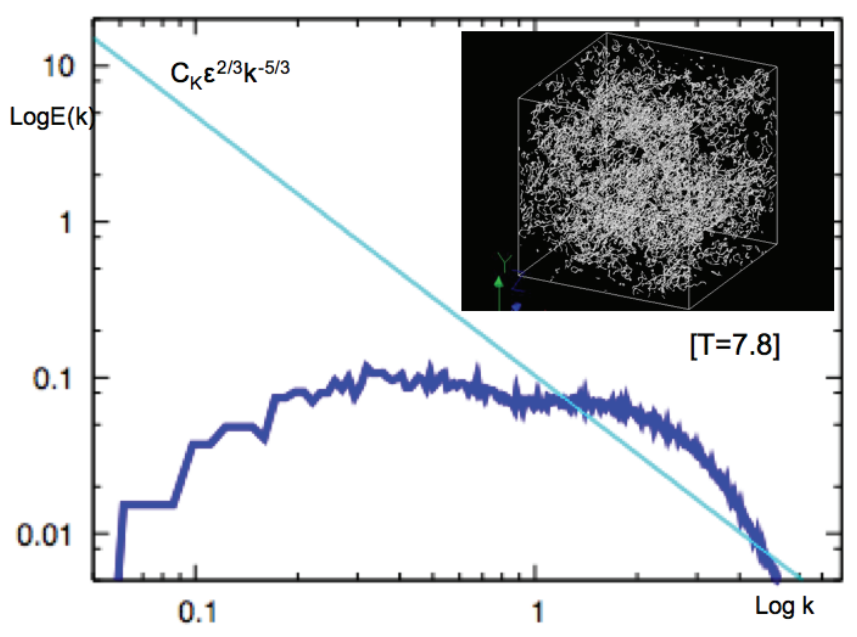

(c) $\mathrm{T}=7.8$

Fig. 1 The incompressible energy spectra and a snapshot of vortex lines (in panel) are shown at time $\mathrm{T}=1.2(\mathrm{a}), \mathrm{T}=3.8(\mathrm{~b})$ and $\mathrm{T}=7.2(\mathrm{c})$. The numerical grid size is $512^{3}$.

At the beginning of the time evolution, each cube moves like a lump with a random velocity and collides each other. The collision produce many vortices and then the system change into turbulent state.

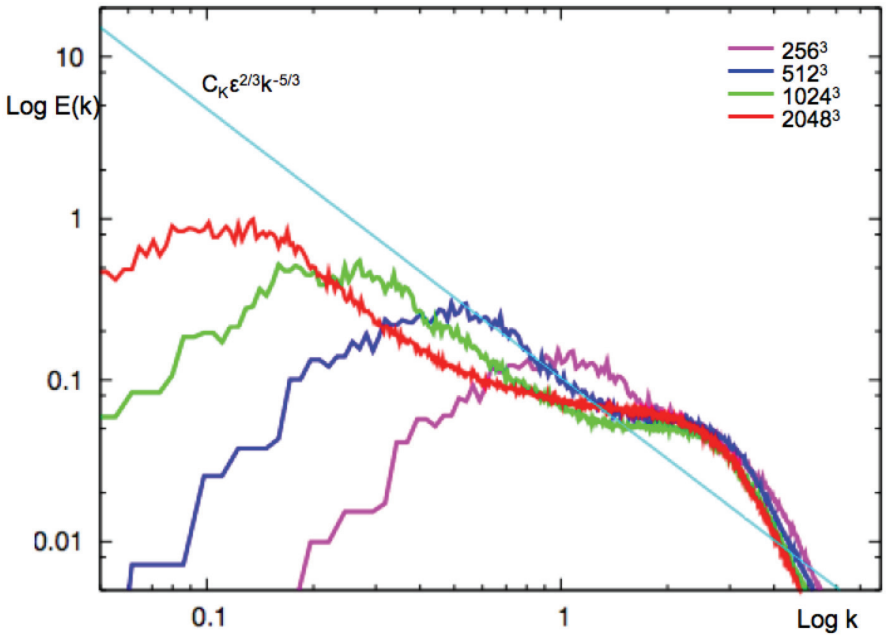

Fig. 2 Simulation results of the incompressible energy spectra $\mathrm{E}_{\text {kin }}^{\mathrm{i}}(\mathrm{k}, \mathrm{t}) . \quad 256^{3}$ :pink-line, $512^{3}$ :blue-line, $1,024^{3}$ :green-line, $2,048^{3}$ :red-line

\section{Numerical Scheme}

In our numerical simulation, we use a fast fourier transformation under the cyclic boundary condition. The spatial grid points are chosen as $512^{3}, 1,024^{3}$ and $2,048^{3}$, and spatial mesh is $\Delta x=1 / 8$. The numerical scheme of time evolution is a 2nd-order symplectic integrator with $\Delta \mathrm{t}=0.0025$.

In each time step, higher wavenumber components, $|\mathbf{k}|=$ $\left[\mathrm{k}_{\mathrm{x}}{ }^{2}+\mathrm{k}_{\mathrm{y}}{ }^{2}+\mathrm{k}_{\mathrm{z}}{ }^{2}\right]^{1 / 2}>2 \pi / \xi$ are cut off, where $\xi$ is the condensate coherence length. It should be noted that no aliasing error occurred by using the wavenumber cut off and the 2nd-order symplectic method. To compensate the eliminated components, the order parameter $\Psi(\mathbf{r}, \mathrm{t})$ is renormalized in every time step to retain $\mathrm{N}_{\text {tot }}$ to be a constant.

\section{Numerical Results}

\section{Time Evolution}

We show a numerical result of $512^{3}$ case as a typical time evolution of quantized vortex tangle and energy spectrum in Fig. 1. At the beginning, few vortices appear and the major part of the energy spectrum located at lower wavenumber area (Fig. 1(a)). In Fig. 1(b), one finds that the major part of the energy spectrum fits the K41 law. As expected, we also observed tangled vortex bundles clearly demonstrated in the insets of Fig. 1(b). On the other hand, Fig. 1(c) is a typical example of a considerably decayed state, in which the main feature is rather small vortex rings distributed almost equally inside the simulation cubic region. Although we introduce the cut off wavenumber $\mathrm{k}_{\mathrm{c}}=2 \pi / \xi$, it is clear that there are few components which locate at the cut off region in Fig. 1. The K41 law is not affected by the cut off in the present simulation.

\section{Inertial Range}

It is known that, in the isotropic homogeneous normal fluid turbulence, the inertial range becomes large as the system size becomes large. To confirm whether the fact is 


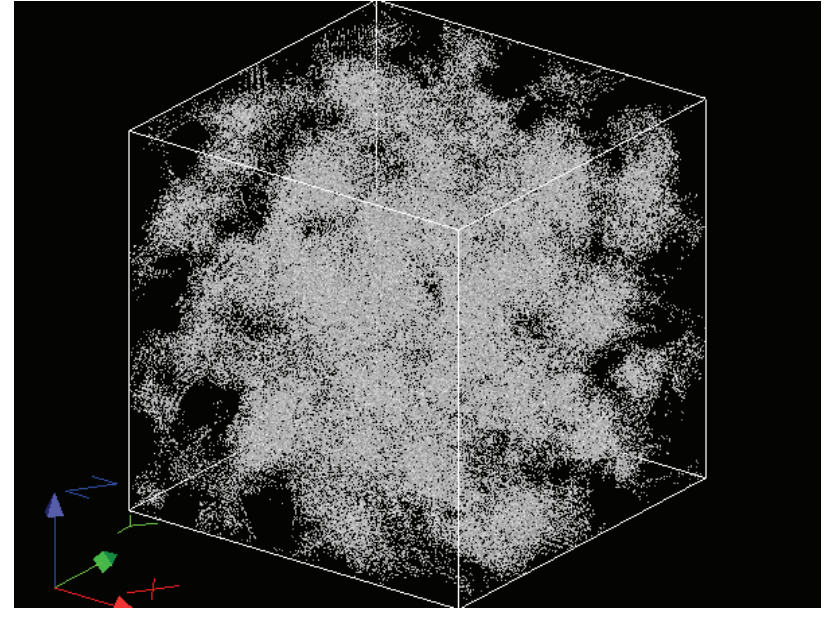

(a)

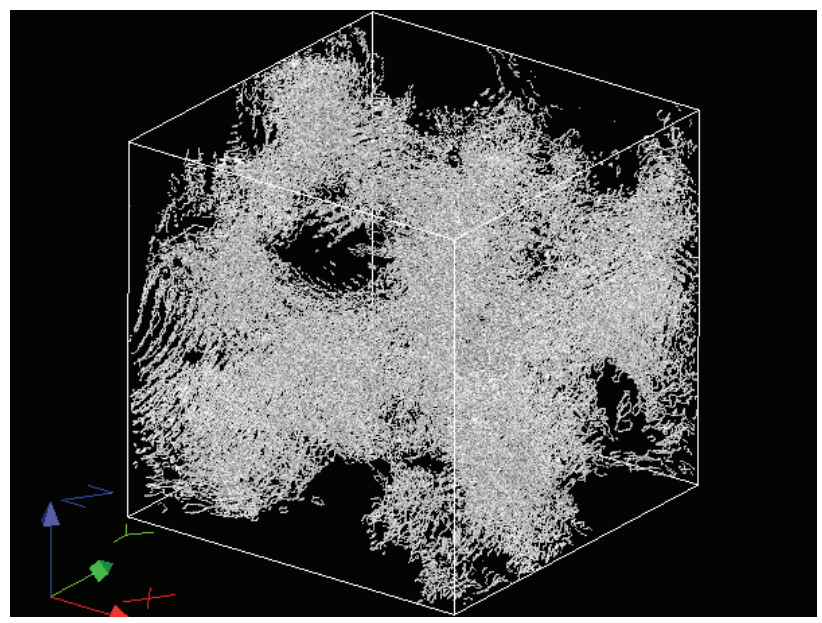

(b)

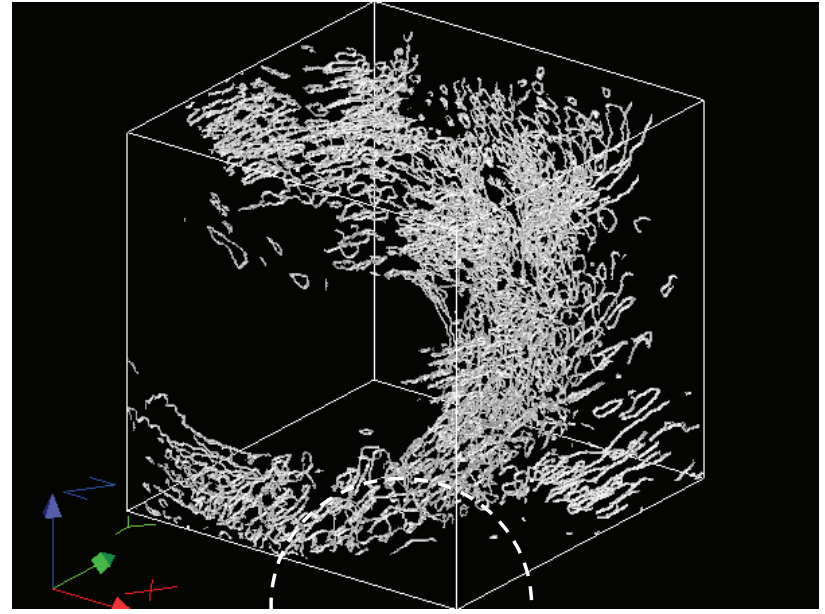

(c)

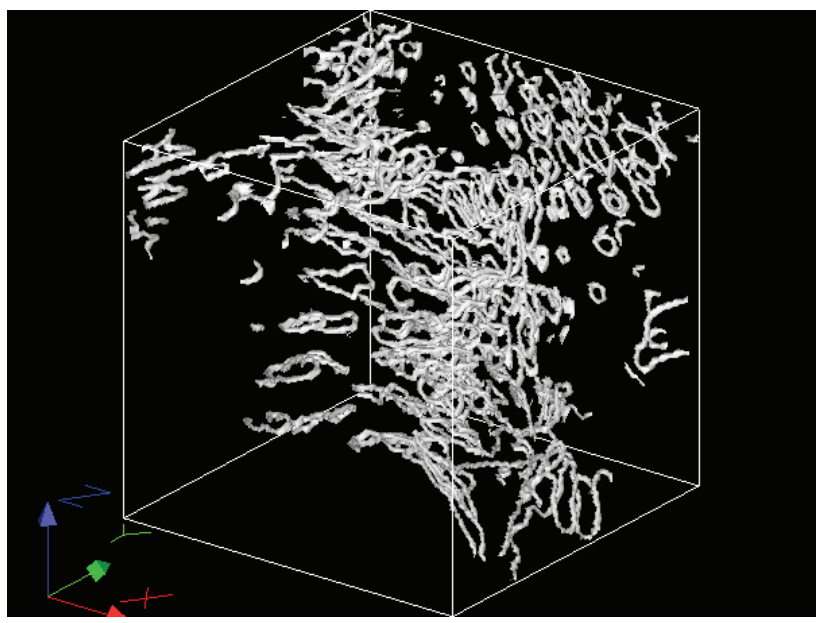

(d)

Fig. 3 A snap shot of vortex lines at the fully developed turbulent state of $2,048^{3}$. The dotted circles represent the zoom regions where vortex distributions are shown subsequently in (a) $\rightarrow(\mathrm{b}) \rightarrow(\mathrm{c}) \rightarrow(\mathrm{d})$.

also true in superfluid case, we investigate the size dependence of the incompressible kinetic energy spectrum $\mathrm{E}_{\mathrm{kin}}^{\mathrm{i}}(\mathrm{k}, \mathrm{t})$. Figure 2 shows an inter-comparison of the incompressible kinetic energy spectrum $\mathrm{E}_{\mathrm{kin}}^{\mathrm{i}}(\mathrm{k}, \mathrm{t})$ among $256^{3}$, $512^{3}, 1,024^{3}$ and $2,048^{3}$ simulations. The K41 scaling is extended to lower $\mathrm{k}$ range with the grid-size increase. This is a clear demonstration of the classical K41 scaling characteristic for the normal fluid turbulence but maintained in the large-scale range of the superfluid turbulence. In each cases, the edge of the inertial range approximately locate at $\sim \mathrm{k}=2 \pi /(\mathrm{L} \Delta \mathrm{x}) 8$. The visible extend of the K41 scaling on $2,048^{3}$ grid is much larger than that in all previous simulations.

In this paper, the dissipation rate of the energy spectrum is roughly estimated by a simplified formula,

$$
\varepsilon=-\frac{\partial}{\partial t} \int_{k}^{\infty} E_{k i n}^{\mathbf{i}}(k, t) d k
$$

From the value of $\varepsilon$, we roughly estimate the Kolmogorov constant $\mathrm{C} \sim 1$ in the $512^{3}$ case.
Figure 3 displays self-similar large structures of tangled vortices in the fully turbulent state. The large-scale vortex bundles in the maximum size, 2,048 ${ }^{3}$, and smaller self-similar tangled structures inside this cubic region in the subsequent figures.

\section{Bottleneck Effects}

In Fig. 2, an important observation is a plateau-like region for - a definite pile-up over the K41 spectrum - a clear manifestation of the energy stagnation. The plateau-like region in larger wavenumber side disturbs the inertial range. That is very different from the classical normal fluid turbulent case. Since the plateau become large with the system size, the inertial range does not simply become large, as is the case of classical normal fluid turbulence.

\section{Conclusion}

We show the resemblance and the difference between the superfluid turbulence and the normal fluid turbulence by numerical simulation of the Gross-Pitaevskii equation. We conclude that the observed essential bottleneck energy ac- 
cumulation has definitely quantum nature (quantization of circulation).

\section{References}

1) W. F. Vinen, R. J. Donnelly, "Quantum Turbulence," Phys. Today, 60, 43 (2007).

2) C. Nore, M. Abid, M. E. Brachet, "Kolmogorov Turbulence in low-Temperature Superflows," Phys. Rev. Lett., 78[20], 3896-3899 (1997), "Decaying Kolmogorov turbulence in a model of superflow," Phys. Fluids, 9[9], 2644-2669 (1997).

3) M. Kobayashi, M. Tsubota, "Kolmogorov Spectrum of Superfluid Turbulence: Numerical Analysis of the Gross-Pitaevskii
Equation with a Small-Scale Dissipation," Phys. Rev. Lett., 94, 065302 (2005), "Kolmogorov Spectrum of Quantum Turbulence," J. Phys. Soc. Jpn., 74, 3248 (2005).

4) J. Yepez, G. Vahala, L. Vahala, M. Soe, "Superfluid Turbulence from Quantum Kelvin Wave to Classical Kolomogrov Cascades," Phys. Rev. Lett., 103, 084501 (2009).

5) V. S. L'vov, S. Nazarenko, "Spectrum of Kelvin-wave turbulence in superfluids," Pisma v ZhETF, 91, 464 (2010).

6) M. Machida, N. Sasa, T. Kano, V. S. L’vov, S. Nazarenko, O. Rudenko, M. Tsubota, "Energy Spectra of Quantum Turbulence : Large-scale Simulation and Modeling," arXiv:1008.3050v1 (2010). 